\title{
Research on Construction and SWRL Reasoning of Ontology of Maize Diseases
}

\author{
Li Ma, Helong Yu, Guifen Chen, Liying Cao, and Yueling Zhao \\ College of Information and Technology Science, Jilin Agricultural University, \\ Chang Chun, Jilin, China \\ mary19801976@sohu.com
}

\begin{abstract}
In this paper, according to the characteristics of maize disease knowledge, OWL DL language was used to build maize diseases ontology, and the reasoning rule of maize diseases was defined by using the expressive ability of SWRL rule language. The author introduced several realizable reasoning functions, and achieved the diagnostic reasoning of maize disease knowledge by Jess inference engine. The results indicated that constructing the maize diseases ontology, and introducing SWRL rule into maize disease ontology provided an effective way for the construction of high-intelligent, shareable and reused maize disease knowledge database and diagnostic rule database.
\end{abstract}

Keywords: ontology of maize diseases, SWRL, SQWRL, Rule reasoning.

\section{Introduction}

Corn is the main grain crop, cultivate area and total output is next to wheat, rice and reside the third. Disease influencing corn production of major natural disasters, annual loss of $6 \% \sim 10 \%$.In recent years, with the replacement of varieties and cultivation techniques of change, the serious diseases happened in the past, have not been solved thoroughly, new disease occur ceaselessly, bring great harm to maize production, prevention and treatment of diseases in maize has become the guarantee of sustainable yield is the key link of Maize[1,2].

At present, the diagnosis and treatment of diseases, mostly based on expert experience, building the database, expert system is used to complete the diagnosis. The traditional knowledge representation methods are difficult to realize complex integrated expert system for the rapid development and the sharing and reusing of demand, recall and precision rate can not be guaranteed. Resulting in the need for a new knowledge representation method to construct the knowledge base, to realize the sharing and reuse between heterogeneous systems[3].

Ontology is the shared conceptual model explicit specification [4], and the definition of ontology for knowledge sharing and reusing the body being applied to artificial intelligence and other related fields become the good knowledge representation tools relatively. However, semantic expression ability of ontology is limited to description logic, can't say the rules of general form, knowledge reasoning ability is limited. The 
SWRL can make up of the shortcomings for ontology modeling technology and ontology language of the OWL, realize the perfect combination of the rules and knowledge[5].

In this paper, use OWL DL language as ontology building language, construction of maize disease ontology database, using SWRL to construct the maize disease related SWRL rules, using the SQWRL query and diagnosis for establishment, to provide reference for the building of sharing and reuse maize disease knowledge base.

\section{The Establishment of Maize Disease Ontology}

\subsection{The OWL Description of Ontology}

Ontology is a description of the relationship between the concept and concept model, through the relationship between the concepts to the semantic description. Ontology contains five basic modeling primitives; those are concepts, relations, functions, axioms, instances. OWL (Web Ontology Language) is the standard of language W3C recommendation to describe Ontology. The description of the OWL for knowledge mainly from the following several aspects, (1) Concept: on behalf of the Concept of field set, Concept of each Concept is a object class, (2) Instances: and that individuals, is a special case of the concept, or the bottom of the object; (3) Properties: attribute hierarchical relationships between the concept is set, used to describe and definition and concept of ontology and examples, it including data attributes and object attributes, object attributes value range is the definition of the class, and the data attribute values can be Boolean, byte, double etc data types[6,7].

\subsection{The Concept and Attribute of Maize Disease Ontology}

Maize diseases mainly include: Top rot, leaf blight, helminthosporium maydis, brown spot, stalk rot, dwarf mosaic disease, rough dwarf disease, Gray Leaf Spot, black silk ear disease, bacterial wilt, General describe as "leaf blight disease onset of parts are in leaves, leaf sheaths or bracts, the early symptoms of leaves on a brown spindle spot", ," Corn Gray Leaf Spot happens in growth period, leaf symptoms early maturity for small spot necrosis, hazel, has faded green halo, Disease spot is not transparent", and so on. Terminology Description of Maize Diseases has a lot of, for example, diseases related terms have names, site of disease onset, symptoms, onset period, and incidence portions include the root, stem and leaf, ear, Onset period can be divided into seedling stage, growth period, and maturation.

In this paper, extract maize disease related terms, including the name of disease, pathogenic sites, the symptom and onset period, Form 4 top-level classes, including: disease class, describe the name of disease; time class, describe the onset period; symptoms class, describing onset of symptoms; class part, describing onset of parts. After each top level class determined, according to knowledge of top level class, subclasses are built. In class symptoms establishing site symptoms subclasses, including 4 parts: class root, class stem, class ear, class leaf, to describe root, stems, ear, 
leaf disease symptom. In the ontology, the specific name of disease, pathogenesis, symptoms of disease, disease period, are added in the form of an example. Corn Disease ontology concept system as shown in Figure 1.

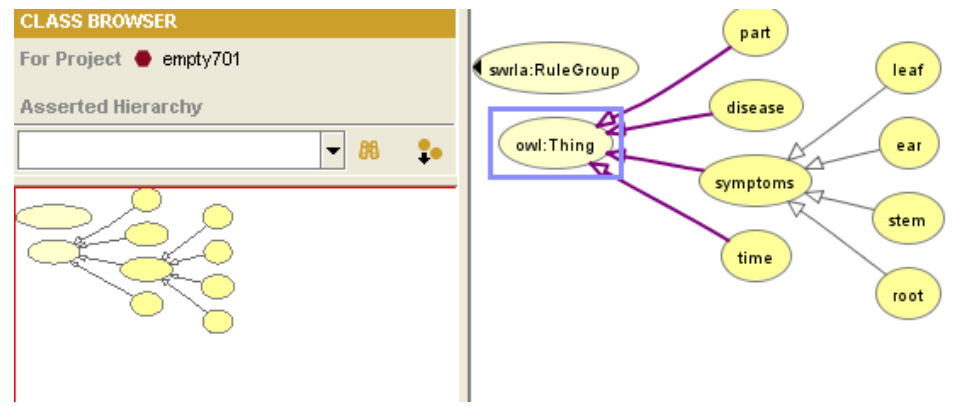

Fig. 1. Corn Disease ontology concept system

After definition of the concept of hierarchical structure, in order to describe the semantic relation between concepts, need to define the attributes of concepts. The object property is used to describe the concepts and concept or instance and instance relations. We also need to define the domain, range, property type and inverse properties as constraint conditions. In this paper, the maize disease ontology set up a total of 5 object properties, results as shown in table 1 .

Table 1. The properties of Maize disease ontology

\begin{tabular}{|c|c|c|c|c|}
\hline Object properties & Domain & Range & description & Inverse properites \\
\hline is_time & time & disease & Onset period & \\
\hline is_part & part & symptoms & Incidence of parts & \\
\hline hassyptom & disease & symptoms & $\begin{array}{l}\text { Onset of } \\
\text { symptoms }\end{array}$ & issyptomof \\
\hline is_disease & thing & disease & $\begin{array}{l}\text { The name of the } \\
\text { disease }\end{array}$ & \\
\hline issyptomof & symptoms & disease & $\begin{array}{l}\text { The symptoms of } \\
\text { the disease }\end{array}$ & hassyptom \\
\hline
\end{tabular}

In the ontology, the specific name of disease, pathogenesis, symptoms of disease, disease period, is added with the form of examples. Adding example of maize disease 10 , example of the root symptoms 3, examples of the stems root symptoms 3 , examples of the stem symptoms 6, examples of the ear 5, examples of the leaf symptoms 16, examples of onset period 3, examples of sites 4, and determine the constraint axiom. Corn Disease ontology instances and constraints are as shown in figure 2 . 


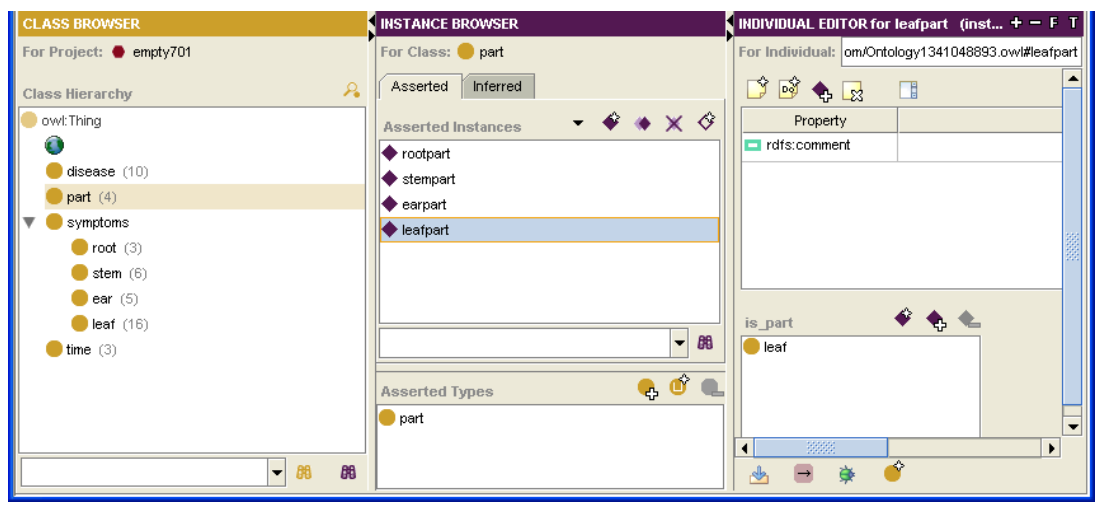

Fig. 2. The ontology constraints and examples

\subsection{The Establish of SWRL Rules}

SWRL (Semantic Web Rule Language) is a strong ability to express the rule description language based on OWL, Which is based on OWL language OWL DL and OWL Lite.

SWRL extends the OWL axiom set, joined the horn clause form rules, rules and the OWL knowledge base can be combined. SWRL consists of Imp, Atom, Variable and Build-in, totally four parts. Imp SWRL rules,Imp is the SWRL rules part, Consists of body and head. Head and body are made up of atom conjunctive composition, variables of atom record in the variable. Built-in is a modular component of SWRL, which records the SWRL can refer to logical relations, namespace is swrlb[8].

SQWRL (Semantic Query-Enhanced Web Rule 13 Language) is based on the SWRL ontology query language, with build-in predicate thesaurus as expansion point; use build-in to build search rule [9].SQWRL includes Core Operators and Collection Operators two parts, Core Operators query through sqwrl: select, Collection Operators support more advanced search functions, to provide advanced grouping and aggregation function. Because SQWRL use standard SWRL syntax, does not change the semantics of SWRL, so in the SWRL editor SQWRL rules can be added and modified, through SQWRL Query Tab convert the SWRL to a query effectively. SQWRL supports OWL class description, by inference can be constrained examples, can be used to retrieve the ontology contains the implicit information, and expanded the build-in predicate lexicon, enriched the numerical, logic comparison predicate and statistical functions, very convenient to use.

\section{$3 \quad$ Maize Disease of Reasoning Based on SWRL}

\subsection{Reasoning Rules Construction}

SWRL Editor is the expansion of Protégé -OWL, so that the user can edit rules at Protégé, more convenient and intuitive. SWRL Editor can verify SWRL rules of basic syntax and semantics test, to ensure that each rules in grammar is correct. 
In the framework of SWRL, conditional of constraints is established in Atom, the rules established in the Imp[10], we will use two kinds of constraints:

$\mathrm{C}(\mathrm{x}): \mathrm{C}$ is $\mathrm{OWL}$ description $\mathrm{P}(\mathrm{x}, \mathrm{y}): \mathrm{P}$ is a property of $\mathrm{OWL}$, wherein $\mathrm{X}$, y can be variables, OWL individuals or OWL data value.

In the established maize disease ontology, extracted specific reasoning elements disease involved according to concepts of ontology, and according to the element forming rules of inference. Corn Disease ontology rules are mainly based on symptoms to infer the disease, thus in the extraction of corn disease symptom knowledge, must ensure that the knowledge is correct, taking the typical, main symptoms. In this paper, take 10 kinds of common diseases in maize diseases as an example, writing maize diseases rules. Firstly, extract maize disease symptom. Secondly, the associated symptoms are added to the body, to ensure that the rules used in all atoms are from the body or property, that is to say not introduce new variables in the rules, this is SWRL defined by a safety limit. The extracted portions of the rules are as follows:

Rule1- Top rot: disease (? X) $\wedge$ hassymptom (? X, Upper Leaf Chlorosis) $\wedge$ hassymptom (? X, the blade edge or tip withered yellow stripe generated) $\wedge$ hassymptom (? X, internode transverse visible Brown lesion) ^hassymptom (? X, ear top is changed to pink_ $) \wedge$ is_time $($ ? $X$, chengshuqi $) \wedge$ is_time $($ ? $X$, shengzhangqi $)$ $\wedge$ is_time $($ ? X, shengzhangqi) $\wedge$ is_part $($ ? X, Jing $) \wedge$ is_part $($ ? $X, S U I) \wedge$ is_part $($ ? X, $\mathrm{ye}) \rightarrow$ is_disease ( $\mathrm{x}$, dingfubing?)

Rule2- helminthosporium maydis:disease (? X) ^hassymptom (? X, early immersion shape tan or reddish brown spots then $) \Lambda$ hassymptom (? X, severe ear rot seed black mildew) $\wedge$ is_time $(? \mathrm{X}$, chengshuqi) $\wedge$ is_time $(? \mathrm{X}$, miaoqi) $\wedge$ is_time $(? \mathrm{X}$, is_part (shengzhangqi)? $\mathrm{X}$, ye) $\wedge$ is_part $(? \mathrm{X}, \mathrm{SUI}) \rightarrow$ is_disease $(? \mathrm{x}$, helminthosporium maydis)

In the diagnostic rules, "disease" is the class in the ontology, "is_disease", "hassymptom", "is_time", "is_part" is the object attribute.

\subsection{SWRL Rule Reasoning Process}

Reasoning rules can be carried out reasoning and searching after building the ontology, but SWRL Editor itself does not perform any reasoning, need the help of other inference tool for reasoning, this paper uses Jess (Java expert system shell) inference engine to do the reasoning[11]. Jess is the CLIPS (C Language Integrated Production System) inference engine based on Java language, its core is composed of 3 parts of fact base, rule base, inference engine and the use of production rules as basic knowledge expression model. Jess supports the forward and backward reasoning, is a good performance of the open type inference machine. Base on advantages above, use Jess as the reasoning engine in the SWRL reasoning rules.

Maize Disease ontology is build using OWL language, then to construct the corresponding rules of SWRL to make OWL reasoning ability inadequacy, thereby forming an ontology knowledge base, including rule library and fact library two aspects. Through Jess and SWRL editor is integrated, the diseases of Maize OWL 
ontology and rules of SWRL converted to Jess fact library and Jess rule library, calling the Jess engine for reasoning, and push reasoning result into the knowledge library, and format new knowledge. The reasoning mechanisms of SWRL are as shown in Figure 3.

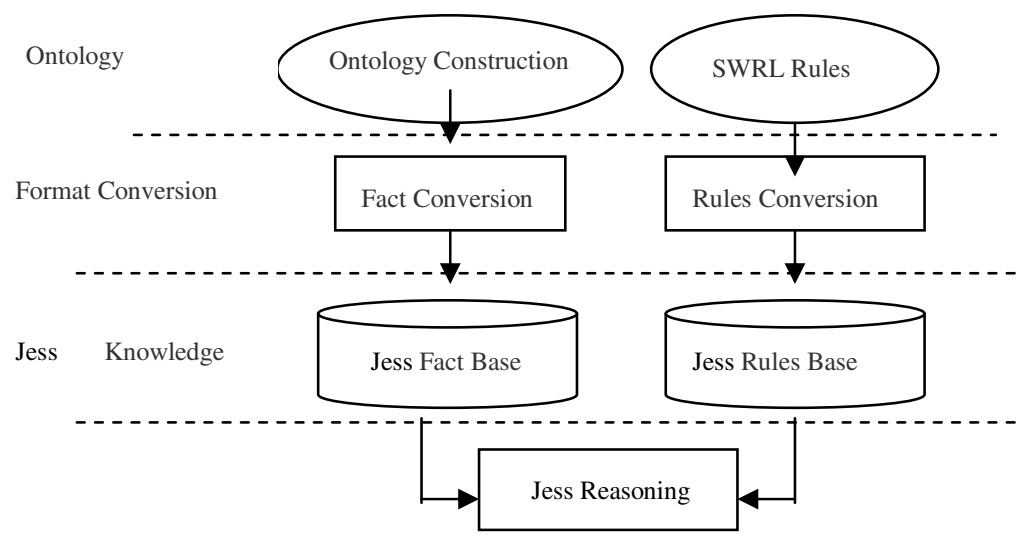

Fig. 3. The reasoning mechanism of SWRL

\subsection{Maize Disease Ontology SWRL Diagnostic Reasoning}

This paper uses ontology as a knowledge organization method, using SWRL Editor to preparation rules, through the SQWRL query, to mainly achieve the following function of maize diseases diagnosis:

known symptoms of the disease, diagnosis disease name

For example, rule: disease (? X) $\wedge$ hassymptom (? X, Upper Leaf Chlorosis $) \wedge$ hassymptom (? X, the blade edge or tip withered yellow stripe generated) $\Lambda$ hassymptom (? X, internode transverse visible Brown lesion) $\rightarrow$ sqwrl: select (? X)

Known symptoms of Upper Leaf Chlorosis and leaf edges, yellow stripes or tip withered, internodes transverse visible brown lesion tissue, through the SQWRL query, receive the diagnosis conclusion: may be suffering from top rot.

Known disease name, to infer the possible symptoms

For example, rule: hasSymptom (top rot,? Y) ^is_part (?x, leaf) $\rightarrow$ sqwrl: $\operatorname{select}($ ? y)

Known as the top rot disease on site for leaf, through the SQWRL query, receive leaf symptoms

(3) According to the different condition, realize the query function. For example, rule:

(1) is_part (? x, leaf) $\rightarrow$ sqwrl:select (?x) Through the SQWRL query, the query of the ontology all may occur in the leaf disease of.

(2) is_time (?x, Seedling stage) $\rightarrow$ sqwrl: select(? x) Through the SQWRL query, get possible disease of corn seedling. 


\section{Conclusion}

This paper studied the maize disease ontology construction, and use SWRL and SQWRL to describe the rules of maize disease diagnosis, completes the ontology in a specific subject application, and practice it. Many domestic scholars carried out through SWRL disease diagnosis [12], but they are using SWRL known disease symptoms to diagnose the disease name, and must meet all of the symptoms can be diagnosed with the disease. In this paper, on the basis of SWRL, using the SQWRL extension reasoning function, rich in disease diagnosis function, improve disease diagnosis.

But corn disease diagnosis is a very complicated process, which involves elements of multiple and complex, the same disease at a different position and different period have been demonstrated, so if you want to achieve accurate maize disease diagnosis function, will add more judgment conditions, and will continue to refine the concept. The next step work include: (1) to further improve maize disease ontology, concept refining; (2) research on SWRL semantic extension, solving predicate logic operation and between a predicate logic or operational problems, further improve the semantic rules legibility and universality, reducing the maintenance cost and rules, to simplify the process of reasoning, facilitates users to complete reasoning.

Acknowledgments. Funding for this research was provided by youth fund of Jilin agricultural university (NO.201133,) and the development of science and technology plan projects of Jilin province (NO. 201101114), Jilin provincial projects(NO.201248), The word bank project(No. 2011-Z20), Hall of Jilin province science and technology science and technology support program(20110237,20120282).

\section{References}

1. Jie, C.: Maize disease diagnosis and prevention and control. Shield Press (2002)

2. Chen, L., Wang, L.: Probabilistic neural network in the maize leaf department of the application of the disease recognition. Farm Machinery Research (2011)

3. Ma, L., Yu, H., Wang, Y., Chen, G.: The Knowledge Representation and Semantic Reasoning Realization of Productivity Grade Based on Ontology and SWRL. In: Li, D., Chen, Y. (eds.) CCTA 2011, Part I. IFIP AICT, vol. 368, pp. 381-389. Springer, Heidelberg (2012)

4. Studer, R., Benjamins, V.R., Fensel, D.: Knowledge engineering: principles and methods. Data \& Knowledge Engineering (1998)

5. Liu, C.: The Research of Corn Disease and Pest Prevention and Cure Semantic Searching System Based on Ontology. Jilin University (2007)

6. Li, J., Su, X.-L., Qian, P.: The methodology of developing domain ontology. Computer and Agriculture, 7-10 (2003)

7. Wang, X., Wei, Y.-Y.: Research on Construction and SWRL Reasoning of Swine Disease Noumenon. Acta Agriculturae Jiangxi (2012) 
8. Zongnan, S., Zheng, Y.L., Qian, P.: Based on the GuiYu SWRL disease diagnosis knowledge representation and semantic reasoning realize. Journal of Intelligence (2010)

9. Wang, H., Fan, L., Li, Z.-Z.: Semantic Web Service Discovery Based on SQWRL. Microelectronics \& Computer (2010)

10. Ji, Z.-H., Li, C.-H.: Constructing and Strategy Analyzing of Rule System for the Semantic Web Based on SWRL and Jess. Journal of Huaihai Institute of Technology (Natural Sciences Edition), 26-29 (2009)

11. Sandia National Laboratories. JESS, the Rule Engine for the Java Platform (EB/OL) (November 11, 2008), http: / / Herzberg.ca.sandia.gov.JESS (July 12, 2009)

12. Mou, D.M., Fan, Y.: SWRL reasoning mechanism based on the ecg ontology design and implementation. Journal of Intelligence (2010) 